\title{
Meta
}

Journal des traducteurs

Translators' Journal

\section{GRANGER Sylviane et PAQUOT Magali (dir.) (2012) : Electronic Lexicography. Oxford: Oxford University Press, 517 p.}

\section{Guy Lapalme}

Volume 59, numéro 2, août 2014

URI : https://id.erudit.org/iderudit/1027484ar

DOI : https://doi.org/10.7202/1027484ar

Aller au sommaire du numéro

Éditeur(s)

Les Presses de l’Université de Montréal

ISSN

0026-0452 (imprimé)

1492-1421 (numérique)

Découvrir la revue

Citer ce compte rendu

Lapalme, G. (2014). Compte rendu de [GRANGER Sylviane et PAQUOT Magali

(dir.) (2012) : Electronic Lexicography. Oxford : Oxford University Press, 517 p.]

Meta, 59(2), 457-459. https://doi.org/10.7202/1027484ar

Ce document est protégé par la loi sur le droit d'auteur. L'utilisation des services d’Érudit (y compris la reproduction) est assujettie à sa politique d'utilisation que vous pouvez consulter en ligne.

https://apropos.erudit.org/fr/usagers/politique-dutilisation/
Cet article est diffusé et préservé par Érudit.

Érudit est un consortium interuniversitaire sans but lucratif composé de l’Université de Montréal, l'Université Laval et l'Université du Québec à Montréal. Il a pour mission la promotion et la valorisation de la recherche. https://www.erudit.org/fr/ 


\section{DOCUMENTATION}

\section{Comptes rendus}

Granger Sylviane et Paquot Magali (dir.) (2012): Electronic Lexicography. Oxford: Oxford University Press, $517 \mathrm{p}$.

Ce livre regroupe 20 communications (en anglais) écrites par des experts mondiaux de la lexicographie. Tous les articles traitent des conséquences, heureuses et moins heureuses, pour les lexicographes et les utilisateurs de dictionnaires, de l'utilisation de l'informatique et d'Internet. Certains articles s'intéressent aux rédacteurs (nouveaux outils de gestion, analyse de corpus) alors que d'autres insistent sur les nouveaux modes de consultation et les usages qu'ils induisent chez les lecteurs. En plus des dictionnaires classiques, on présente aussi de nouveaux types de dictionnaires plus spécialisés qu'il n'aurait probablement jamais été rentable de publier sous forme papier, mais que l'informatique rend viables à cause du coût de diffusion relativement faible et du fait qu'Internet permet d'en élargir la diffusion.

Comme dans tous les recueils de ce type, la qualité et l'intérêt, au moins pour l'auteur de ce compte-rendu, diffèrent passablement d'un article à l'autre, mais l'ensemble est captivant et devrait l'être pour toute personne qui s'intéresse à la lexicographie moderne.

Même si les articles sont autonomes, les références ont été regroupées dans une bibliographie de plus de 40 pages, en plus de deux index: des sujets traités et des auteurs cités. Les dictionnaires discutés et les URL cités dans un article sont toutefois indiqués à la fin de l'article plutôt qu'être regroupés en fin de volume, un choix discutable. Les multiples références entre les différents articles du livre ne sont malheureusement indiquées que par (XYZ, this volume) plutôt que par un numéro de chapitre ou un numéro de page.

Étant donné le sujet, il peut sembler paradoxal de publier sous format papier plutôt que de façon électronique. Cette évaluation est basée sur la lecture de la version papier noir et blanc ${ }^{1}$ dans laquelle certaines copies d'écran sont malheureusement difficiles à lire: les caractères y sont trop petits, la copie ayant été réduite à la largeur de la page et les couleurs sur l'écran sont difficiles à distinguer en tons de gris. Un système interactif est parfois difficile à apprécier avec une suite de copies d'écran. Heureusement, lorsque des URL publiques sont fournies, on peut suivre à l'écran les exemples au fur et à mesure de la lecture de l'article.

Le premier chapitre est une introduction à l'ensemble des articles qui présente brièvement les auteurs et résume leur contribution. Les chapitres suivants ont été divisés en trois grandes parties de longueur à peu près égale ( 6 articles) : la lexicographie à un moment décisif, des dictionnaires innovants et des études sur les usagers des dictionnaires électroniques.

La première partie réunit le «Who's who» de la lexicographie en langue anglaise: un ex-éditeur en chef des dictionnaires anglais chez Oxford (Patrick Hanks), l'éditeur en chef des dictionnaires Macmillan (Michael Rundell) et un éditeur de logiciel commercial d'analyse de corpus (Adam Kilgariff) utilisé par toutes les grandes maisons d'édition de dictionnaires.

J'ai particulièrement apprécié le chapitre 2 qui présente le point de vue d'un éditeur de dictionnaire sur la lexicographie automatisée. Après avoir fait un tour d'horizon sur l'introduction des outils informatiques dans la création de dictionnaires commerciaux, il présente des réflexions très éclairantes sur l'économique du remplacement des dictionnaires papier par des versions électroniques et sur le rôle des lexicographes maintenant qu'on dispose d'outils d'analyse de corpus et d'extraction d'information. Il traite également du besoin de disposer de très grands corpus (plus d'un milliard de mots) afin de permettre aux lexicographes de séparer les utilisations anecdotiques des usages réels des mots dans la langue courante. Ce dernier point de vue est corroboré dans le chapitre 4 par Patrick Hanks sur l'impact des corpus sur la lexicographie en prenant pour exemple l'étude des collocations qu'il compare avec ce qu'on retrouve dans FrameNet et Wiktionary. L'étude sur le verbe throw est, de l'avis même de l'auteur du chapitre, un peu longuette, mais il est très intéressant de suivre cet exemple jusqu'au bout.

Les chapitres 3 et 5 se limitent à des descriptions d'outils, certes intéressants pour qui n'en a jamais entendu parler, mais leurs auteurs livrent peu de réflexions quant à leur impact, et ne comparent pas ces outils entre eux. Le sixième chapitre par Sven Tarp met la table pour une intéressante discussion théorique sur les différences entre la lexicographie papier et électronique, mais après avoir bien argumenté pendant dix pages qu'une telle théorie existe, il s'arrête sur un court panorama d'outils électroniques. Quant au septième 
chapitre, il traite de la lexicographie pour des langues peu dotées en prenant des exemples de langues d'Afrique du Sud; après avoir analysé les dictionnaires existants pour ces langues et les avoir qualifiés de coquilles vides, l'article ne porte guère de réflexion sur leur (in)adéquation.

La deuxième partie présente de nouveaux types de dictionnaires qu'il n'aurait pas été possible d'imaginer sous une forme papier.

Le chapitre qui m'a le plus surpris dans cette partie est le chapitre 12 qui porte sur la lexicographie basée sur WordNet. Après avoir donné les bases et les concepts de WordNet (la section 12.2 est la meilleure description d'une page de WordNet que j'ai lue), il présente un dictionnaire pour la langue serbe construit au moyen de la traduction à la main des synsets de WordNet et de la création de nouveaux lorsque les traductions ne pouvaient se faire directement. On y a ensuite ajouté des innovations typiquement web comme la recherche dans les corpus, des mécanismes d'externalisation ouverte (crowdsourcing) et des illustrations obtenues via Flickr. L'auteur termine avec des réflexions très intéressantes sur des extensions éventuelles pour des idiomes et des collocations. Le chapitre 13 compare les entrées du Wiktionary et celles des dictionnaires construits par des experts. Cette étude est très intéressante, car elle porte sur plusieurs langues et fait des comparaisons qualitatives et quantitatives (en termes de nombre d'entrées). Même si les auteurs sont assez critiques des connaissances des usagers pour identifier certaines subtilités des sens des mots différents, ils croient que le fait que le processus d'édition soit ouvert peut rendre le modèle Wiktionary compétitif avec les dictionnaires construits de manière plus traditionnelle.

Le chapitre 11 présente un tout nouveau modèle de dictionnaire ${ }^{2}$ : le DiCoInfo est avant tout une base de données spécialisée d'informations lexicales consultable uniquement par le web. La base trilingue (français, anglais et espagnol) porte sur les mots et les expressions dans le domaine de l'informatique. Les auteurs expliquent que l'encodage des collocations à l'aide de fonctions lexicales permet de faciliter la recherche de l'expression la plus appropriée dans un contexte d'usage donné (traduction ou apprentissage d'une langue seconde). Ils présentent différentes implantations qui ont été faites de ces idées et les résultats d'une évaluation auprès d'usagers. Le chapitre 14 présente les problèmes soulevés par la construction de dictionnaires électroniques pour le langage des signes, en particulier comment trouver des modes d'indexation appropriés et intuitifs. Les auteurs rapportent leur expérience pour le danois.

Le chapitre 8 présente les résultats d'une étude effectuée auprès d'usagers afin de déterminer comment adapter l'interface d'un dictionnaire. Si l'idée est louable, le texte est malheureusement une suite de captures d'écran pratiquement illisibles, car trop pâles et en caractères trop petits, ce qui est dommage pour un article sur la convivialité. Le chapitre 9 traite d'un outil pour aider à rédiger des textes didactiques en anglais langue seconde et il souffre du même problème de présentation que le précédent en se limitant à une suite de captures d'écran difficiles à lire; ceci est d'autant plus dommage que, contrairement à l'article précédent, l'accès au site est limité aux étudiants et au personnel de l'Université Catholique de Louvain. Le chapitre 10, rédigé par un des directeurs de l'ouvrage, présente un dictionnaire bilingue basé sur des textes scientifiques qui vise des lecteurs, des traducteurs et des linguistes pour qui ils ont développé des sections particulières de leur site web. Même si les captures d'écran sont lisibles dans le livre, il est plus intéressant de suivre en visitant le site qui est en accès libre.

La troisième partie du livre est consacrée aux usagers des dictionnaires électroniques.

Le chapitre 17 a été le plus surprenant pour moi, car il discute d'usages inavoués de dictionnaires de poche ou de sources discutables qui sont souvent des listes de mots sans contexte d'utilisation ni vérification d'attestation de leur usage. Le chapitre 19 présente les caractéristiques idéales qu'on aimerait retrouver dans un dictionnaire électronique. Ces caractéristiques sont ensuite utilisées pour comparer les définitions associées à cinq mots dans des dictionnaires papier et sur Internet. L'auteur conclut que les dictionnaires gratuits sur Internet sont surtout utiles à cause des multiples liens qu'ils donnent vers d'autres ressources, mais qu'ils peuvent être aussi une source de bruit, car ils amènent parfois trop d'information, n'étant pas limités par le nombre de pages.

Le chapitre 20 est le compte-rendu d'une analyse statistique de questionnaires remplis par quelques centaines de personnes en 2010 sur leur utilisation et préférences pour les dictionnaires en ligne. Les résultats sont analysés avec beaucoup (peut-être même un peu trop) de minutie. La méthodologie est bien décrite et pourrait sûrement servir d'exemple pour d'autres études du même type. Les résultats sont très intéressants et même un peu contre-intuitifs : le multimédia et la possibilité d'adapter l'interface à l'usager ne sont pas jugés importants quoiqu'il faudrait peut-être éduquer les usagers pour qu'ils puissent mieux en tirer parti.

Le chapitre 15 décrit une série d'études portant sur les usagers des dictionnaires. Le chapitre 16 donne une liste de points dont il faudrait tenir compte dans le développement d'une interface pour un dictionnaire électronique. Le chapitre 18 est consacré à l'utilisation des dictionnaires 
électroniques par les traducteurs. Ce sujet, très intéressant pour les lecteurs de cette revue, est malheureusement plutôt une liste de souhaits souvent partiellement réalisés par certains dictionnaires ou systèmes présentés dans le livre. L'auteur n'aborde malheureusement pas le problème plus fondamental de leur intégration dans un poste de travail qui permettrait d'améliorer la productivité d'un traducteur.

Dans l'ensemble, j'ai beaucoup apprécié ce livre qui apporte plusieurs éclairages nouveaux et des points de vue différents, parfois contradictoires entre les articles. Ces réflexions forcent à remettre en question certains aspects du métier des lexicographes qui, comme tous les travailleurs du savoir, doivent s'adapter à l'arrivée de l'informatique en revoyant les façons de faire traditionnelles. Les dictionnaires électroniques changent aussi pour les utilisateurs la manière de consulter les dictionnaires.

Même si les éditeurs sont francophones et travaillent à l'Université Catholique de Louvain comme professeurs d'anglais langue étrangère, on ne trouve aucun article rédigé par un éditeur de dictionnaire français, peut-être parce que comme le souligne Patrick Hanks (p. 62):

For some languages, e.g. French, there is no major corpus-based dictionary at all. In other languages, the situation is rather different: for example, major corpus-based dictionaries of languages as different as Danish, Modern Greek, and Malay have been published.

Pourtant plusieurs dictionnaires électroniques existent pour le français comme en témoignent les actes, seulement électroniques, eux, de la $4^{\mathrm{e}}$ Journée québécoise des dictionnaires (Cormier 2012).

Guy Lapalme Département d'informatique et de recherche opérationnelle Université de Montréal, Montréal, Québec, Canada

\section{NOTES}

1. Ce document est également disponible auprès d'Oxford Scholarship Online: www.oxfordscholarship.com/view/10.1093/ acprof:oso/9780199654864.001.0001/ acprof-9780199654864

2. L'auteur de ce compte-rendu est un collègue des auteurs de cet article et il a déjà collaboré à ce projet.

\section{RÉFÉRENCE}

Cormier, Monique (2012): $4^{\text {e }}$ Journée québécoise des dictionnaires - Actes du Colloque international: Du papier au numérique: la mutation des dictionnaires:

http://moniquecormier.ca/4e-journee-quebecoise-des-dictionnaires/actes/
Orozco-Jutorán, Mariana (2012): Metodología de la Traducción Directa del Inglés al Español. Materiales didácticos para traducción general y especializada. Granada: Editorial Comares, 1-293.

This book is for translator trainees to develop general and specialised competences for translation from English (L2) to Spanish (L1). A literal translation of the title is 'A Methodology of Direct Translation from English to Spanish' and its purpose is to give students a method with which to approach any kind of translation brief. Translator trainers working from English into other languages may also find inspiration for programming courses, teaching units and tasks, as well as a wide variety of source texts covering genres in different fields and modalities of translation. Published in 2012, it is already being used in several translation academic faculties in Spain and has been tested for several years at the Universitat Autònoma de Barcelona (UAB) where the author, Mariana Orozco-Jutorán has been teaching translation since 1996. In the introduction she acknowledges special debt to Amparo Hurtado Albir for introducing her to translator training and a translation-task-based approach.

This is a practical manual in which translation theory is taught implicitly. Students can come to their own conclusions about translation, learning inductively through a series of tasks that make them think and reach systematic solutions. Emphasis is put on the translation process, identifying problems and strategies for solving them within a specific translation brief. There are no ready-made solutions as students are encouraged to develop their own potential and processes in accordance with their own experience, knowledge and skills. Although the book is solidly based on recent theoretical approaches in translation studies, linguistics, information and communication technologies, documentation and terminology, these approaches are implicit in the different teaching units and specific theories and authors are not mentioned.

The teaching methodology follows the guidelines recommended by the European Higher Education Area to develop transversal and specific competencies. A task-based approach is used to promote the acquisition of translation competence, including all the different sub-competences. Once again, this underlying structure is implicit and the author avoids using the jargon that so often makes these guidelines so opaque to outsiders. However, each teaching unit also has a final section called 'Reflections' that summarises the main concepts addressed in the unit. 